\title{
SEPARABILIDADE DE AGREGADOS RECICLADOS PROVENIENTES DE RESÍDUOS DE CONSTRUÇÃO E DEMOLIÇÃO DE DIFERENTES ORIGENS
}

\author{
C. ULSEN ${ }^{1}$; H. KAHN ${ }^{1}$; S. C. ANGULO ${ }^{2}$; V. M. JOHN² e H. HAWLITSCHEK \\ ${ }^{1}$ Depto. de Eng. de Minas e de Petróleo da Escola Politécnica - Universidade de São Paulo. ${ }^{2}$ Depto. de Eng. de \\ Construção Civil da Escola Politécnica - Universidade de São Paulo. carina@lct.poli.usp.br
}

Artigo submetido em novembro/2013 e aceito em dezembro/2013

http://dx.doi.org/10.15628/holos.2014.1785

\begin{abstract}
RESUMO
Resíduos de construção e demolição constituem a maior parcela dos resíduos sólidos urbanos. A proibição de sua disposição em aterros, a redução na oferta de agregados naturais somada ao aumento de sua demanda, e a crescente exigência para o desenvolvimento de aplicações sustentáveis para tais resíduos impulsionaram o desenvolvimento de aplicações para este material. Diferentes estratégias foram estabelecidas para melhorar a qualidade dos agregados reciclados (reduzir sua porosidade), no entanto, a liberação das fases presentes é raramente discutida na literatura. Neste trabalho, são apresentados os resultados de um estudo de separabilidade realizado em agregados reciclados
\end{abstract}

provenientes de resíduos de construção e demolição de diferentes origens. Os resultados mostram que a proporção de material mais denso aumenta nas frações mais finas e indicam maior liberação entre as fases presentes, especialmente para as frações abaixo de 12,7 $\mathrm{mm}$. Os produtos obtidos diferem expressivamente entre $\mathrm{si}$, independentes de sua origem, o que pode ser confirmado pelos teores de aglomerantes, perda ao fogo e pela sua composição mineralógica. Tais diferenças refletem diretamente nas propriedades físicas dos agregados reciclados (absorção de água, porosidade e resistência mecânica).

PALAVRAS-CHAVE: liberação, agregados reciclados, resíduos de construção e demolição.

\section{SEPARABILITY STUDIES ON RECYCLED AGGREGATES FROM DIFFERENT CONSTRUCTION AND DEMOLITION WASTES}

\section{ABSTRACT}

Construction and demolition waste constitute the largest portion of municipal solid waste. The prohibition of their disposal in landfill, the increasing demand for aggregates, the environmental restriction for aggregates extraction and the growing requirement for the development of sustainable applications for construction and demolition waste, stimulated the development of applications for this material, particularly in the concrete, which is by far the most important construction material. Different strategies have been established to improve the quality of recycled aggregates (reduce their porosity), such as optimized comminution, heat treatment and others. However, the liberation between the present phases is seldom discussed in the literature. To develop an efficient
\end{abstract}

recycling process, able to separate the cement paste from the aggregates, it is first necessary to know the liberation behavior of the cement paste under crushing along the particles size distribution. In this paper, it is discussed the results of separability studies by heavy liquid separations in recycled aggregates from construction and demolition waste from different sources. The results show that the proportion of heavy products increases specially on factions bellow $12.7 \mathrm{~mm}$. The products differ notably among them, which can be confirmed by the content of binder, loss on ignition and mineralogical composition. These differences reflect directly on recycled aggregates physical properties (water absorption, porosity and mechanical resistance).

KEYWORDS: liberation curves, recycled aggregates, construction and demolition waste. 


\section{INTRODUÇÃO}

Entende-se por resíduos de construção e demolição (RCD) como sendo a parcela mineral dos resíduos gerados durante o ciclo de vida das construções civis, o que inclui blocos, concreto e outros materiais de alvenaria, solo, rocha, madeira, forros, argamassa, gesso, telhas, pavimento asfáltico, entre outros (BIOCYCLE, 1996; LEVY, 1997; ZORDAN, 1997). Assim, é um material que possui uma grande variabilidade, sendo esta uma das dificuldades no seu processo de reciclagem.

Reciclar resíduos de construção e demolição como agregados é necessário para fechar o ciclo de produção na construção civil (HENDRIKS, 2004), evitando o consumo elevado de agregados naturais, assim como o uso de áreas de aterros que estão escassas no meio urbano. Tanto no Brasil quanto no exterior, esses agregados reciclados são majoritariamente utilizados em aplicações de menor exigência técnica, tais como nivelamento de terrenos e sub-bases de pavimentos (Mueller, 2003; ANGULO, 2005). Parte desta limitação técnica está associada à composição do RCD, particularmente no que se refere ao conteúdo de pasta de cimento porosa aderida nas partículas (BARRA e VAZQUEZ, 1998; DE JUAN e GUTIERREZ, 2009).

Diversos estudos propõem rotas mais elaboradas de reciclagem, como o emprego de choque térmico seguido de moagem (TATEYASHIKI, SHIMA et al., 2001), britagem por ondas de choque (LINSS e MUELLER, 2004), ou simplesmente realizar mais etapas de britagem (NAGATAKI, GOKCE et al., 2004). Contudo, para se desenvolver um processo de reciclagem eficiente, capaz de separar a pasta de cimento aderida nos agregados, primeiro é necessário conhecer o comportamento de liberação da pasta de cimento sob a britagem ao longo da granulometria. A liberação ocorre quando fases diferentes são eficientemente separadas, desapegadas umas das outras. Se a liberação não for realizada com sucesso a separação torna-se ineficiente, o que significa que é necessária um grau de liberação razoável para se obter uma boa separação (GAUDIN, 1932). No caso dos agregados reciclados, a liberação de materiais, que depende muito do tamanho das partículas, raramente é discutida nesses estudos.

Neste trabalho é apresentado o resultado de um estudo de separabilidade realizado em agregados de RCD reciclados, obtidos por britagem de resíduos de construção e demolição provenientes de diferentes origens. Como a pasta de cimento é mais porosa que o agregado natural (granito), investigou-se o uso da densidade como propriedade diferenciadora. Os resultados confirmam que a densidade é uma propriedade diferenciadora e demonstram a influência da redução do tamanho das partículas na liberação das fases.

\section{MATERIAIS E MÉTODOS}

\subsection{Amostras}

Foram caracterizadas três diferentes amostras de resíduos de construção e demolição britados (agregados reciclados), os quais foram obtidos por amostragem em duas usinas de reciclagem do Estado de São Paulo, sendo uma na capital (usina de Itaquera - duas amostras) e uma no interior (Vinhedo).

A classificação visual dos resíduos em duas classes de acordo com a sua cor (cinza predominância de materiais cimentícios, e vermelho - resíduo com grande proporção de materiais 
cerâmicos) era utilizado em ambas as usinas. A amostragem foi realizada em três produtos de duas usinas: Itaquera: cinza e vermelho, Vinhedo: vermelho, denominadas IC-C, IT-V e VI-V.

Uma descrição detalhada das usinas é apresentada por Ulsen (ULSEN, 2006) e sintetizada a seguir:

a) Usina de Itaquera: usina de médio porte localizada na antiga pedreira Itaquera, zona leste da cidade de São Paulo, com processamento de materiais cerâmicos e cimentícios. Empregava as seguintes operações unitárias: seleção manual (ou catação - antes e após a britagem), cominuição em britador de impacto (capacidade de $100 \mathrm{t} / \mathrm{h}$ ), extração de metais por eletroímã após a britagem e peneiramento a seco em 40, 20 e 4,8 $\mathrm{mm}$.

b) Usina de Vinhedo: usina de pequeno porte localizada na cidade de Vinhedo, a $80 \mathrm{~km}$ de São Paulo com processamento predominantemente de materiais cerâmicos (cerca de $80 \%$ ). As operações unitárias empregadas eram: seleção manual para remoção de contaminantes e da fração metálica, cominuição em britador de mandíbulas (capacidade de $8 \mathrm{t} / \mathrm{h}$ ) e peneiramento a seco em 12,7; 9,5 e 4,8 mm.

\subsection{Procedimento experimental}

As atividades laboratoriais são sumarizadas abaixo:

Homogeneização e amostragem: As três amostras obtidas foram homogeneizadas e amostradas em pilha alongada. Ao final, foram retirados cerca de $80 \mathrm{~kg}$ de cada amostra para os estudos de separabilidade.

Peneiramento: As amostras foram peneiradas em meio úmido em peneiras com aberturas nominais de 19,1 a 0,15 mm, gerando seis frações granulométricas.

Separabilidade por líquidos densos: Cada fração granulométrica foi submetida a separações sequenciais em líquidos densos nas densidades de 1,7; 1,9;2,2 e 2,5 g/cm3. Ao final do ensaio foram obtidos cinco produtos para cada fração granulométrica: $d<1,7 ; 1,7<d<1,9 ; 1,9<\mathrm{d}<2,2$; $2,2<\mathrm{d}<2,5$ e $\mathrm{d}>2,5$ (densidade em $\mathrm{g} / \mathrm{cm}^{3}$ ).

Caracterização dos produtos: Determinação da perda ao fogo a 1050 ㄷ $\mathrm{C}$ por $1 \mathrm{~h}$. Estimativa do teor de aglomerantes por lixiviação ácida em $\mathrm{HCl}$ a 20\%; os aglomerantes solúveis reagem com o ácido e são quantificados pela diferença de massa final e inicial da amostra (QUARCIONI e CINCOTTO, 2006). Estimativa da composição mineralógica por meio de análises semiquantitativas por difratometria de raios X (método RIR - Reference Intensity Ratio), método do pó, em equipamento marca PANalytical, modelo X'Pert PRO com tubo de Cu e detector X'Celerator.

Caracterização das propriedades físicas: Para as frações de agregados graúdos (>4,8 $\mathrm{mm})$, foi determinada a absorção de água segundo o método descrito pela norma NBR 9937 (ABNT, 1987) de agregados convencionais. Resumidamente, o método envolve a determinação da condição de superfície saturada seca, que corresponde à condição em que a partícula contém água dentro dos poros acessíveis, mas não há água disponível em sua superfície. A importância da determinação da absorção de água e/ou porosidade deve-se ao fato de esta estar inversamente relacionada à resistência mecânica dos agregados, ou seja, quanto maior a porosidade/absorção de água, menor sua resistência (ANGULO, 2005). 


\section{RESULTADOS}

As curvas de separabilidade por densidade, com valores acumulados nos produtos afundados, são apresentadas na Figura 1.

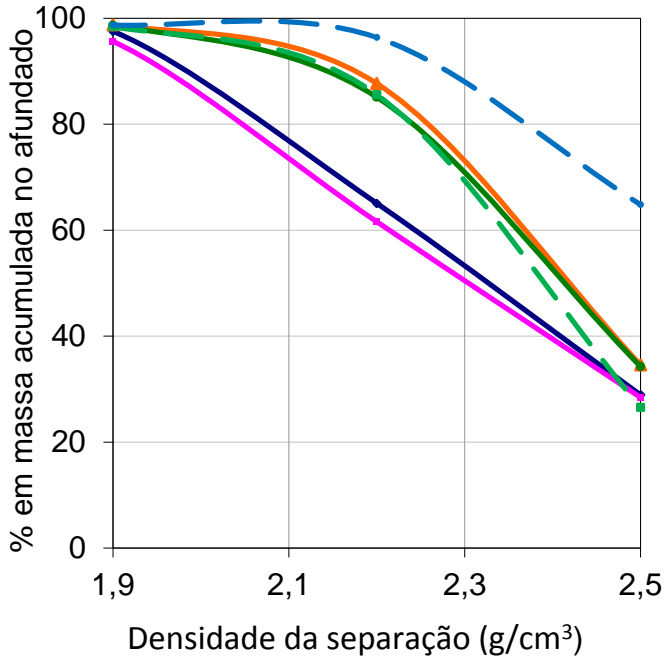

IT-C

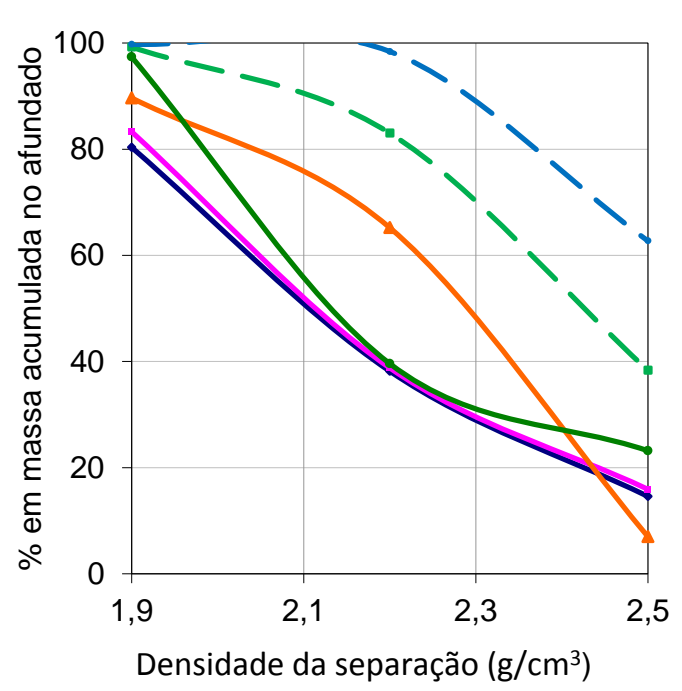

VI-V

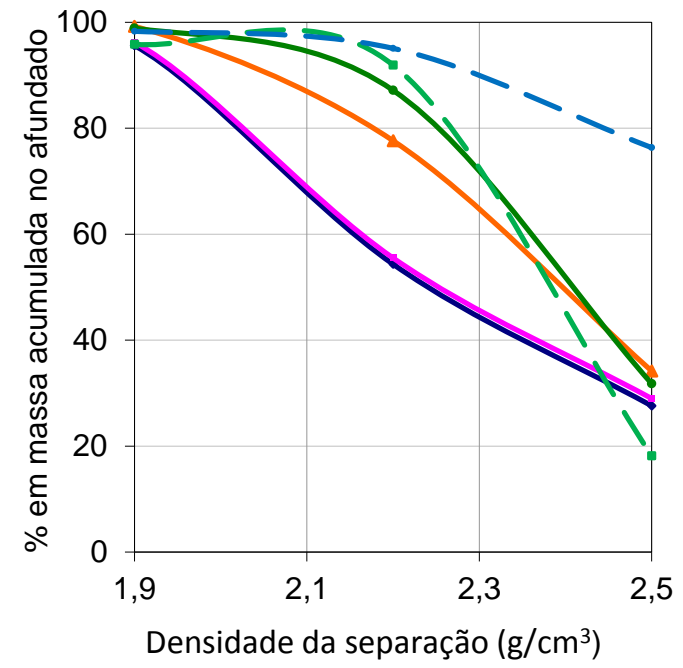

IT-V

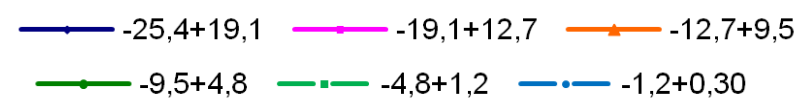

$\%$ em massa acumulada nos produtos de densidade

\begin{tabular}{|c|ccc|ccc|ccc|}
\hline $\begin{array}{c}\text { Fração } \\
\text { (mm) }\end{array}$ & \multicolumn{3}{|c|}{ IT-C } & \multicolumn{3}{|c|}{ IT-V } & \multicolumn{3}{|c|}{ VI-V } \\
\hline $\mathbf{2 1 , 9}$ & $\mathbf{> 2 , 2}$ & $\mathbf{> 2 , 5}$ & $>\mathbf{1 , 9}$ & $\mathbf{> 2 , 2}$ & $\mathbf{> 2 , 5}$ & $\mathbf{> 1 , 9}$ & $\mathbf{> 2 , 2}$ & $\mathbf{> 2 , 5}$ \\
\hline $\mathbf{- 2 5 , 4 + 1 9 , 1}$ & 97,5 & 65,1 & 28,9 & 95,6 & 54,3 & 27,6 & 80,4 & 38,2 & 14,6 \\
\hline $\mathbf{- 1 9 , 1 + 1 2 , 7}$ & 95,6 & 61,6 & 28,4 & 96,2 & 55,5 & 29,0 & 83,3 & 38,8 & 15,9 \\
\hline $\mathbf{- 1 2 , 7 + 9 , 5}$ & 98,8 & 87,7 & 34,6 & 99,2 & 77,6 & 34,2 & 89,7 & 65,2 & 7,0 \\
\hline $\mathbf{- 9 , 5 + 4 , 8}$ & 98,7 & 85,1 & 34,3 & 98,9 & 87,1 & 31,8 & 97,4 & 39,6 & 23,2 \\
\hline $\mathbf{- 4 , 8 + 1 , 2}$ & 98,4 & 85,6 & 26,6 & 95,9 & 91,9 & 18,2 & 99,0 & 71,6 & 29,9 \\
\hline $\mathbf{- 1 , 2 + 0 , 3}$ & 98,7 & 96,4 & 64,8 & 98,3 & 95,1 & 76,3 & 99,5 & 96,4 & 48,3 \\
\hline
\end{tabular}

Figura 1 - Curvas de separabilidade por densidade.

A distribuição em massa acumulada nos produtos afundados mostra que as frações acima de 12,7 $\mathrm{mm}$ apresentam menor proporção de material afundado na separação em líquido com densidade de $2,2 \mathrm{~g} / \mathrm{cm} 3$. Essa proporção aumenta nas frações mais finas, indicando maior liberação da fração mineral de interesse (agregados naturais pré-existentes). As frações intermediárias, abaixo de 12,7 e acima de 1,2 $\mathrm{mm}$ apresentam comportamento similar nas amostras de Itaquera e diferente entre si na amostra de Vinhedo, enquanto que na fração abaixo de $1,2 \mathrm{~mm}$, quase a totalidade da amostra encontra-se acima de $2,2 \mathrm{~g} / \mathrm{cm} 3$.

Os três diferentes agregados apresentam comportamentos semelhantes na separação por densidade para cada intervalo granulométrico, sendo que o VI-V tem maior quantidade de material 
leve $(<1,9 \mathrm{~g} / \mathrm{cm} 3)$ na fração graúda $(>4,8 \mathrm{~mm})$, chegando até a $20 \%$, contra os $5 \%$ dos agregados de Itaquera.

A liberação da fração mineral de interesse, indicada pela proporção em massa de material nos produtos afundados, é maior nas frações granulométricas mais finas; nesse sentido IT-C e IT-V são muito semelhantes, enquanto VI-V apresenta um produto graúdo bastante diferente.

Produtos graúdos $(>4,8 \mathrm{~mm}$ ) de RCD vermelho apresentam distribuição em massa distinta para as duas instalações de reciclagem, sendo que o produto de Itaquera é de melhor qualidade em relação ao produto de Vinhedo quanto à distribuição de massa nos produtos de maior densidade. Os produtos cinza e vermelho de Itaquera apresentaram a mesma distribuição de massa, não sendo, portanto, a cor, um critério seletivo, dessa forma, a classificação baseada no critério visual não pode ser empregada para a avaliação da qualidade dos produtos.

Uma separação por densidade pode ser empregada com o objetivo de se obter agregados graúdos de melhor qualidade, uma vez que mais da metade da massa destes agregados possui massa específica aparente superior a $2,2 \mathrm{~g} / \mathrm{cm} 3$ e podem ser superiores também quanto aos critérios físicos (menor porosidade e absorção de água e maior resistência).

Os produtos miúdos (frações 4,8-0,3 mm) da separação apresentam distribuição semelhante não importando sua origem ou classificação (cinza ou vermelho). Mais de $80 \%$ da massa destes produtos se encontra nos intervalos de densidade acima de $2,2 \mathrm{~g} / \mathrm{cm}^{3}$, o que permite inferir, num primeiro momento, que proporção de fases porosas nos agregados miúdos pode ser inferior à dos agregados graúdos. Entretanto, ensaios para determinação da massa específica aparente e absorção de água da fração miúda são necessários para confirmar tal hipótese.

Os teores de aglomerantes e a correlação entre estes e a perda do fogo, para os produtos da separação por densidade, são apresentados na Figura 2.
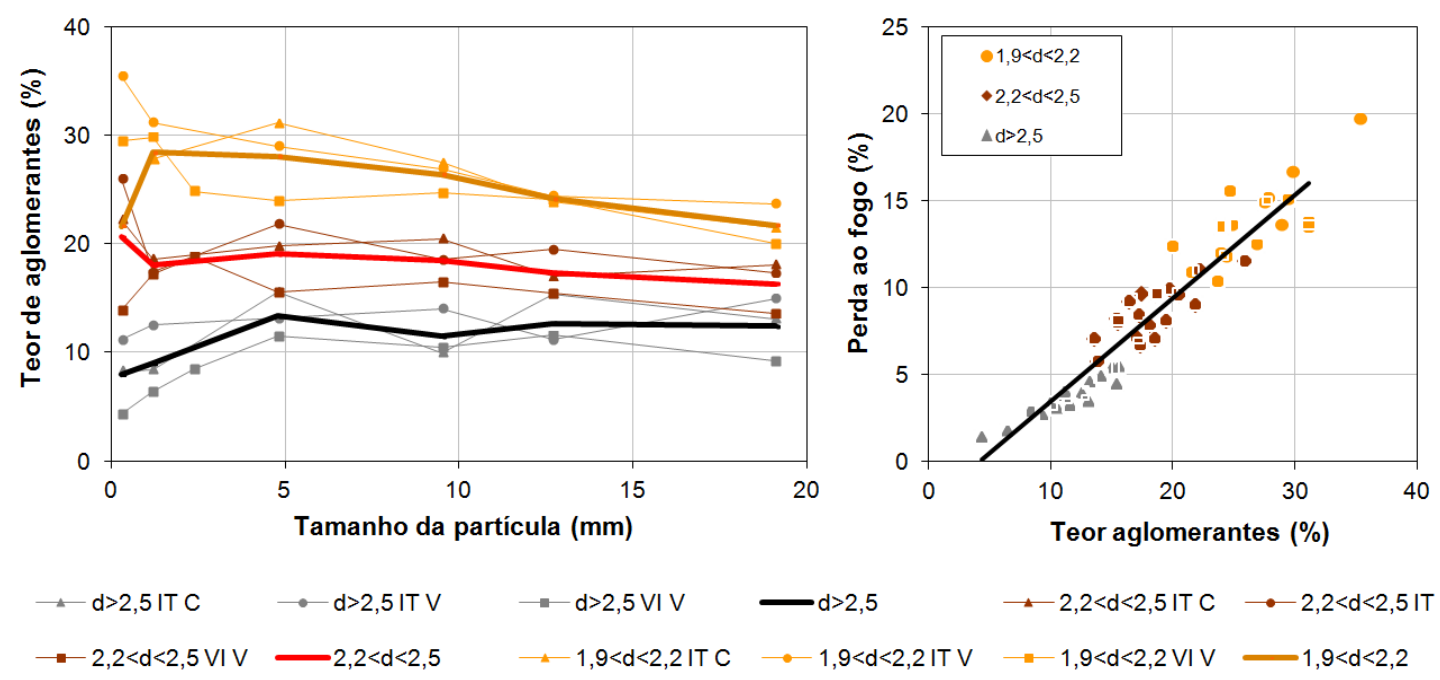

$\mathrm{d}>2,5$
$\ldots 1,9<\mathrm{d}<2,2$ IT V

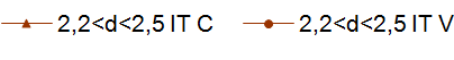

$=1,9<\mathrm{d}<2,2 \mathrm{VIV}-1,9<\mathrm{d}<2,2$

Figura 2 - Teores de aglomerantes estimados para os diferentes produtos da separação por densidade das três amostras (a), Correlação entre perda ao fogo e teor de aglomerantes (b)

Os gráficos mostram claramente que tanto o teor de aglomerantes como a perda ao fogo são essencialmente influenciados pela densidade da separação (Figura 2a) e que quanto maior este valor, menor a perda ao fogo e, logo, menor o teor de aglomerantes. A correlação entre o teor de 
aglomerantes e a perda ao fogo é evidente; o conteúdo de ambos aumenta progressivamente para os produtos com menor massa específica aparente (Figura $2 b$ ).

A composição mineralógica estimada por DRX para os produtos da separação por densidade das três amostras é apresentada na Tabela 1.

Tabela 1 - Estimativa da composição dos produtos (\% massa)

\begin{tabular}{llcccccc}
\hline Produtos & Feldspato & Quartzo & $\begin{array}{c}\text { Feldspato+ } \\
\text { quartzo }\end{array}$ & Mica & Argilominerais & $\begin{array}{c}\text { Pasta de cimento } \\
\text { + carbonatos }\end{array}$ \\
\hline $\mathbf{1 , 7 - 1 , 9}$ & IT-C & 26 & 22 & 48 & 17 & 3 & 32 \\
& IT-V & 26 & 39 & 65 & 22 & 2 & 11 \\
& VI-V & 36 & 16 & 52 & 38 & 10 & 10 \\
& Média & $\mathbf{2 9}$ & $\mathbf{2 6}$ & $\mathbf{5 5}$ & $\mathbf{2 6}$ & $\mathbf{5}$ & $\mathbf{1 8}$ \\
\hline $\mathbf{1 , 9 - 2 , \mathbf { 2 }}$ & IT-C & 36 & 21 & 57 & 25 & 5 & 12 \\
& IT-V & 41 & 31 & 72 & 8 & 6 & 14 \\
& VI-V & 38 & 16 & 54 & 33 & 5 & 9 \\
& Média & $\mathbf{3 8}$ & $\mathbf{2 3}$ & $\mathbf{6 1}$ & $\mathbf{2 2}$ & $\mathbf{5}$ & $\mathbf{1 1}$ \\
\hline $\mathbf{2 , 2 - 2 , 5}$ & IT-C & 65 & 7 & 72 & 21 & 4 & 3 \\
& IT-V & 70 & 12 & 82 & 14 & 3 & 2 \\
& VI-V & 66 & 9 & 75 & 19 & 1 & $\mathbf{3}$ \\
& Média & $\mathbf{6 7}$ & $\mathbf{9}$ & $\mathbf{7 6}$ & $\mathbf{1 8}$ & $\mathbf{3}$ & 3 \\
\hline $\mathbf{2 , 5}$ & IT-C & 79 & 5 & 84 & 13 & 0 & 1 \\
& IT-V & 78 & 4 & 82 & 14 & 3 & 1 \\
& VI-V & 83 & 4 & 87 & 9 & 4 & $\mathbf{2}$ \\
\hline
\end{tabular}

As principais fases identificadas foram a) tectosilicatos provenientes de rochas naturais, b) carbonatos e c) filossilicatos (muscovita/biotita/flogopita, merlinoita e caulinita).

O produto de massa específica aparente menor que $2,2 \mathrm{~g} / \mathrm{cm} 3$ se caracteriza pela presença de carbonatos (essencialmente pasta de cimento), mica e quartzo. Silicatos hidratados das fases cimentícias não foram detectados por difração, mas estão presentes conforme verificado por Angulo (ANGULO, 2005); estes em conjunto com os argilominerais são os principais responsáveis pelos elevados valores de perda ao fogo.

Para os produtos com massa específica aparente superior a 2,2 $\mathrm{g} / \mathrm{cm} 3$ verifica-se um significativo aumento nas proporções de tectosilicatos (quartzo e feldspato) e redução marcante no teor de pasta de cimento, indicando presença de agregados naturais (pedra britada ou fragmentos líticos). Somado a isso, as análises de perda ao fogo confirmam a redução nas proporções de argilominerais e aglomerantes e presença de fragmentos líticos.

Nos produtos concentrados na densidade de separação entre 2,2 e 2,5 g/cm3, os tectosilicatos representam mais de $70 \%$ das fases presentes, enquanto que acima de $2,5 \mathrm{~g} / \mathrm{cm} 3$, esse valor ultrapassa os $80 \%$, atingindo $87 \%$ para a amostra VI-V.

Os valores de absorção de água determinados para as frações de agregados graúdos $(>4,8$ $\mathrm{mm}$ ) são apresentados na Figura 3. Assim como as curvas de separabilidade por densidade e a composição determinada por DRX, a absorção de água também está intimamente associada à densidade da separação. Quanto maior a massa específica aparente do produto (maior proporção de material afundado), menor a absorção de água do agregado reciclado. 
A absorção de água descresce com o aumento da massa específica do agregado, que por sua vez está diretamente relacionada à porosidade do mesmo (ANGULO, 2005). Para os produtos afundados em $2,5 \mathrm{~g} / \mathrm{cm} 3$, a absorção média de água é de $1 \%$.

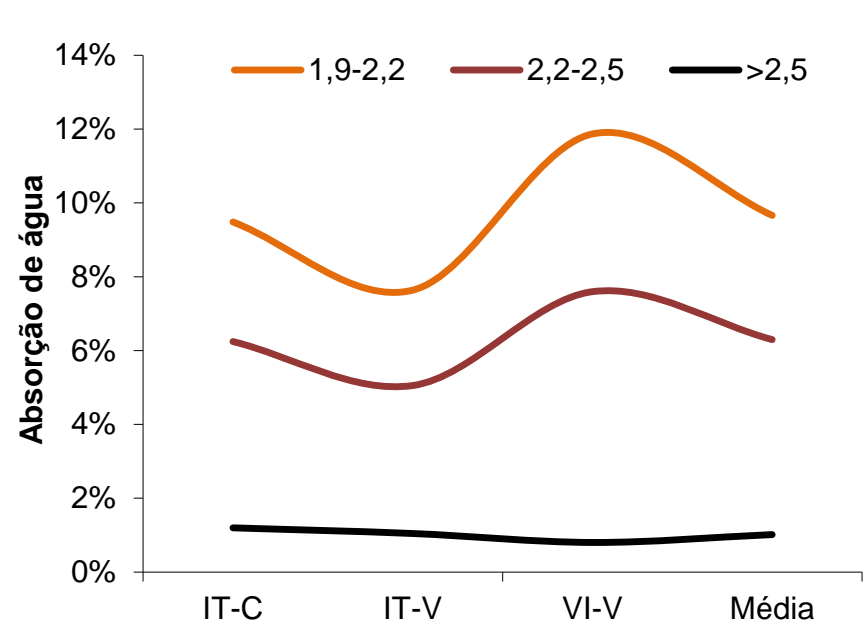

\begin{tabular}{|c|c|c|}
\hline \multicolumn{2}{|c|}{ Produtos $\left(\mathbf{g} / \mathrm{cm}^{3}\right)$} & \multirow{2}{*}{$\frac{\text { ABS (\%) }}{9,5 \%}$} \\
\hline $1,9-2,2$ & IT-C & \\
\hline & IT-V & $7,6 \%$ \\
\hline & VI-V & $11,9 \%$ \\
\hline & Média & $9,7 \%$ \\
\hline \multirow[t]{4}{*}{$2,2-2,5$} & IT-C & $6,2 \%$ \\
\hline & IT-V & $5,1 \%$ \\
\hline & VI-V & $7,6 \%$ \\
\hline & Média & $6,3 \%$ \\
\hline \multirow[t]{4}{*}{$>2,5$} & IT-C & $1,2 \%$ \\
\hline & IT-V & $1,0 \%$ \\
\hline & VI-V & $0,8 \%$ \\
\hline & Média & $1,0 \%$ \\
\hline
\end{tabular}

Figura 3 - Absorção de água para os diferentes produtos da separação por densidade

\section{CONCLUSÕES}

As curvas de separação por densidade mostram que a proporção de material nos produtos de maior densidade aumenta progressivamente nas frações mais finas e indicam maior liberação entre as fases presentes, especialmente para as frações abaixo de $12,7 \mathrm{~mm}$.

As distribuições em massa nos produtos afundados dos resíduos de Itaquera (cinza e vermelho) são bastante semelhantes, no entanto, diferem significativamente do material de Vinhedo (vermelho), indicando que a classificação baseada no critério visual não pode ser empregada para a avaliação da qualidade dos produtos.

Os produtos miúdos da separação apresentam distribuição semelhante, não importando sua origem ou classificação (cinza ou vermelho), onde mais de $80 \%$ da massa destes produtos se encontra nos intervalos de densidade acima de $2,2 \mathrm{~g} / \mathrm{cm}^{3}$.

Os produtos de densidade diferem expressivamente entre si, independentes de sua origem, o que pode ser confirmado pelos teores de aglomerantes, perda ao fogo e pela sua composição mineralógica. Tais diferenças refletem diretamente nas propriedades físicas dos agregados reciclados (absorção de água, porosidade e resistência mecânica).

\section{AGRADECIMENTOS}

Os autores agradecem aos órgãos de fomento FINEP-Habitare (Fundo Verde Amarelo), CNPq, FAPESP (02/08615-0 e 02/00849-1) e às prefeituras das cidades de São Paulo e Vinhedo pelo apoio a realização do presente estudo.

\section{REFERÊNCIAS BIBLIOGRÁFICAS}

1. ABNT: NBR-9937: Agregados - Determinação da absorção e da massa específica de agregado 
graúdo. 1987.

2. ANGULO, S. C. Caracterização de agregados de resíduos de construção e demolição reciclados e a influência de suas características no comportamento dos concretos. 2005. 149 p. Tese (Doutorado) - Escola Politécnica, Universidade de São Paulo, São Paulo.

3. BARRA, M.; VAZQUEZ, E. Properties of concretes with recycled aggregates: influence of properties of the aggregates and their interpretation. In: Sustainable construction: use of recycled concrete aggregate, 1998. London. Proceedings.London: 1998. p. 19-30.

4. BIOCYCLE. Initiating change in C\&D management, Emmaus: 44-48 p. 1996.

5. DE JUAN, M. S.; GUTIERREZ, P. A. Study on the influence of attached mortar content on the properties of recycled concrete aggregate. Construction and Building Materials, v. 23, n. 2, p. 872-877, 2009.

6. GAUDIN, A. M. Principles of mineral dressing. New York: MacGraw-Hill, 1932.

7. LEVY, S. M. Reciclagem do entulho de construção civil para utilização como agregado de argamassas e concretos. 1997. 147 p. Tese (Mestrado) - Escola Politécnica, Universidade de São Paulo, São Paulo.

8. LINSS, E.; MUELLER, A. High-performance sonic impulses - an alternative method for processing of concrete. International Journal of Mineral Processing, v. 74, p. S199-S208, 2004.

9. NAGATAKI, S.; GOKCE, A.; SAEKI, T.; HISADA, M. Assessment of recycling process induced damage sensitivity of recycled concrete aggregates. Cement and Concrete Research, v. 34, n. 6, p. 965-971, 2004.

10. QUARCIONI, V. A.; CINCOTTO, M. A. Optimization of calculation method for determination of composition of hardened mortars of Portland cement and hydrated lime made in laboratory. Construction and Building Materials, v. 20, n. 10, p. 1069-1078, 2006.

11. TATEYASHIKI, H.; SHIMA, H.; MATSUMOTO, Y.; KOGA, Y. Properties of concrete with high quality recycled aggregate by heat and rubbing method. In: JCl, 2001. 2001. p. 61-66.

12. ULSEN, C. Caracterização tecnológica de resíduos de construção e demolição. 2006. 171 p. Tese (Mestrado) - Escola Politécnica, Universidade de São Paulo, São Paulo.

13. ZORDAN, S. E. A utilização do entulho como agregado, na confecção do concreto. 1997. 140 p. Tese (Mestrado) - Faculdade de Engenharia Civil, Universidade de Campinas, Campinas.

14. ROCHA, J. M. P. Definição da Tipologia e Caracterização Mineralógica e Microestrutural dos Itabiritos Anfibolíticos das Minas de Alegria da Samarco Mineração SA MG. . 2008. Tese (Doutorado) - Escola de Engenharia, UFMG, Belo Horizonte.

15. ULIANA, D. et al. Process mineralogy studies of low grade iron ores used in the production of pellet feed In: 10th International Congress for Applied Mineralogy, 1-5 August 2011, 2011. ICAM, 2011. p. 717-724. 